\title{
Liquid crystal alignment on ultrafast laser nanostructured ITO coated glass
}

\author{
Asi Solodar, ${ }^{1}$ Ausra Cerkauskaite, ${ }^{2}$ Rokas Drevinskas, ${ }^{2}$ Peter G. Kazansky, ${ }^{2}$ Ibrahim Abdulhalim ${ }^{1}$ \\ 1. Ben Gurion University of the Negev, Department of Electro-Optics Engineering and The Ilse Katz. Institute \\ for Nanoscale Science and Technology, Beer Sheva 84105, Israel \\ 2. Optoelectronics Research Centre, University of Southampton, Southampton, SO17 1BJ, United Kingdom
}

Liquid crystal (LC) devices are widely used as building blocks of many electro-optical systems including linear polarization rotators, dynamical wave plate retarders, and pixilated devices for displays, spatial light modulators, and tunable filters [1]. Precise alignment of the LC molecules is required for high quality components. The anisotropic nature of LC molecules allows them to align on solid surfaces. This can be achieved either due to physicochemical interaction such as photo-alignment on surfaces using polarized blue light or due to the elastic interaction when aligned along nanogrooves created by mechanical rubbing or lithography techniques [2]. Although numerous methods enabling the manufacturing of LC devices have been reported, the technological flexibility and precision remains a problem.

We propose a direct-write ultrafast laser nanostructuring as an alternative method capable of fabricating anisotropic surface elements. One of the most fascinating aspects of this technique is the ability to induce tunable structures with subwavelength periodicities that generate form birefringence [3]. Such engineered surfaces can be implemented as a host substrate for controlled organization of various materials such as LC molecules. In this study we demonstrate the LC alignment on femtosecond laser nanostructured ITO coated glass. Nine regions of laser-induced nanostructures are depicted in Fig. 1(a). Each column was fabricated under different pulse energy, while the orientations of the anisotropy at each row was changed from horizontal to diagonal and to vertical in order to achieve different alignment directions.

(a)

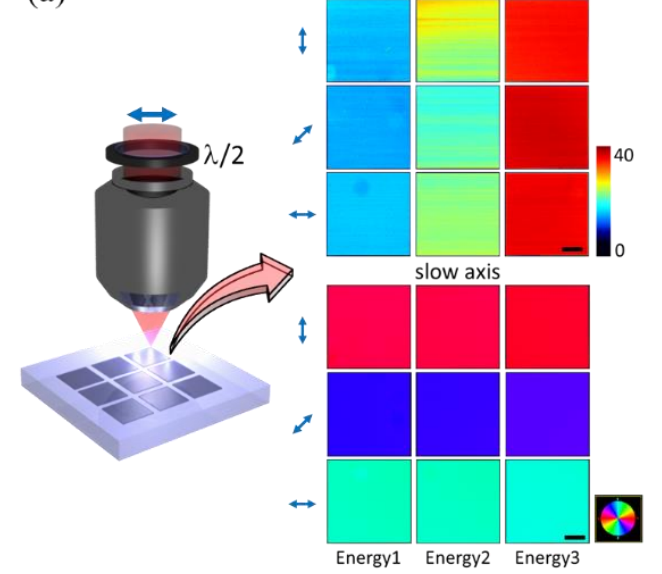

(b)

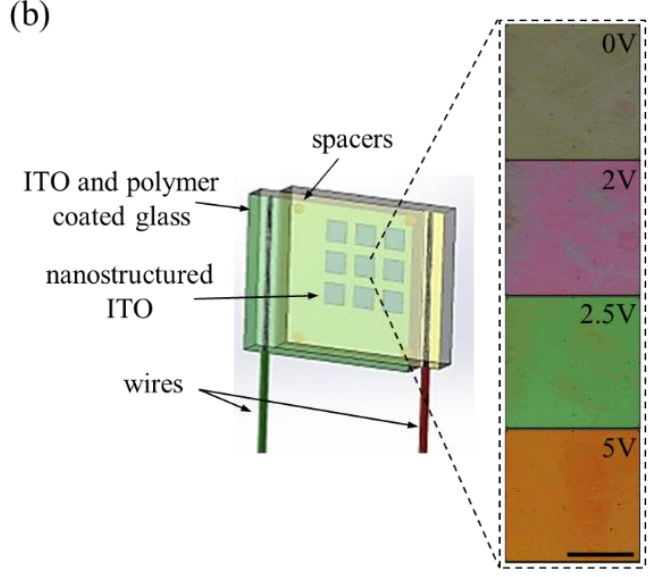

Fig. 1. (a) Femtosecond laser nanostructuring of ITO coated glass towards the fabrication of anisotropic surface elements. Nine regions were imprinted under different writing conditions. The orientation (blue arrows) of linearly polarized incident laser beam was controlled by rotating the half-wave plate $(\lambda / 2)$. The retardance and its corresponding slow axis were imaged using quantitative birefringence measurement system. (b) Schematic of the constructed device. Inset shows polarization microscope images of a central region at different voltages for $45^{0}$ twisted mode. Scale bars are $0.5 \mathrm{~mm}$.

The glass substrate with nanostructured ITO was attached to the additional ITO coated glass which was spincoated with a photoaligning polymer film (ROLIC). After baking and irradiation with polarized UV light the substrates were separated by ten microns spacers. Then the gap was filled with E-7 LC by capillary suction. Finally, two electrodes were connected to the substrates (Fig 1(b)). The cross-polarized reflection images of the device as a function of voltage at $1 \mathrm{kHz}$ is shown in Fig. 1(inset). The results clearly demonstrate LC alignment, and the LC direction follows the nanostructures orientation. This new LC alignment approach can have a great potential for building LC based high precision photonic devices.

\section{References}

[1] D. K. Yang and S. T. Wu, "Fundamentals of Liquid Crystal Devices" (Wiley, New York, 2006).

[2] I. Abdulhalim, "Liquid crystal active nanophotonics and plasmonics: from science to devices," J. Nano Photonics 6, 061001 (2012).

[3] R. Drevinskas, M. Beresna, J. Zhang, A. G. Kazanskii, P. G. Kazansky, "Ultrafast Laser-Induced Metasurfaces for Geometric Phase Manipulation," Adv. Opt. Mat. 5, 1600575 (2017). 